\title{
Rendering Physical Effects in Tabletop Controls
}

\author{
Malte Weiss \\ RWTH Aachen University \\ 52056 Aachen, Germany \\ weiss@cs.rwth-aachen.de
}

\author{
Christian Remy \\ University of Zurich \\ 8050 Zurich, Switzerland \\ remy@ifi.uzh.ch
}

\author{
Jan Borchers \\ RWTH Aachen University \\ 52056 Aachen, Germany \\ borchers@cs.rwth-aachen.de
}

\begin{abstract}
We introduce dynamic physical properties as an additional degree of freedom for passive tabletop controls. Using electromagnetic actuation, we manipulate attributes of tangibles on the fly, such as perceived weight, spring resistance, friction, and latching. We describe our actuation concepts, prototypes, and measurements showing that magnetic fields can change physical effects in a linear way. Controlled experiments reveal that participants can tactually distinguish four rendered resistance levels of a button prototype and easily detect dynamic detents in a continuous slider. Finally, we describe how adjustable physical properties in tangibles can enhance tabletop interaction.
\end{abstract}

\section{Author Keywords}

Tangible user interfaces, interactive tabletops, actuation, haptics, physical properties.

\section{ACM Classification Keywords}

H.5.2 Information Interfaces and Presentation: User Interfaces-Haptic $\mathrm{I} / \mathrm{O}$.

\section{General Terms \\ Human Factors, Experimentation, Measurement}

\section{INTRODUCTION}

Tangible user interfaces on interactive tabletops provide rich physical affordances and haptic feedback. Using the table's rear or top projection they can change their look on the fly. The complexity of these tangibles has evolved significantly in recent years, from rigid bodies (e.g., Reactable [5]) to multi-element widgets, such as knobs and sliders that can be used in a variety of applications (e.g., SLAP [16]).

Most tabletop tangibles are passive controls. Usually, they are made of low-cost material, are easy to build, and do not contain complex electronics. Thus, they are suitable for rapid prototyping. Using appropriate tools, such as a laser cutter, building a widget is a matter of less than an hour.

Permission to make digital or hard copies of all or part of this work for personal or classroom use is granted without fee provided that copies are not made or distributed for profit or commercial advantage and that copies bear this notice and the full citation on the first page. To copy otherwise, or republish, to post on servers or to redistribute to lists, requires prior specific permission and/or a fee.

CHI 2011, May 7-12, 2011, Vancouver, BC, Canada.

Copyright 2011 ACM 978-1-4503-0267-8/11/05...\$10.00.

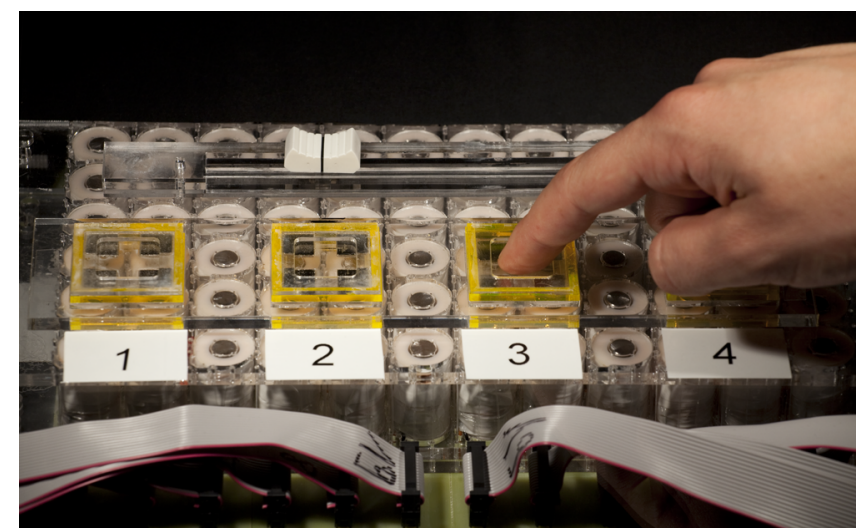

Figure 1. Magnetic actuation allows designers to change physical properties of passive tangible controls. Our test setup includes four buttons with adjustable resistances and a slider with dynamic detents.

While the table's projection allows to change the look of a tangible dynamically, its feel is usually fixed when the physical object is assembled. However, the physical properties of such a control strongly influence the user experience. Swindells et al. [14] show that various parameters in a motor-actuated knob, such as the intensity of friction, inertia, and detents, have a high impact on affective responses and target acquisition times. Changing these properties after the construction of a tabletop control is difficult.

In this paper, we present techniques to render physical properties in tabletop widgets using electromagnetic actuation. While keeping the advantages of low-cost passive controls, our approach allows designers to rapidly prototype physical properties without rebuilding the tangibles, and to change these attributes on the fly. Our studies prove the concept of mapping electromagnetic force to physical properties of tabletop tangibles, such as weight, friction, spring resistance, and detents.

\section{RELATED WORK}

The simulation of dynamic physical properties has gained much attention in the field of Haptic Rendering [13]. The PHANToM interface [7] and the Pantograph [11] emit force vectors on the user's fingertips to simulate the haptic perception of real objects. Although these devices are capable of producing complex haptic feedback in virtual environments, their applicability on interactive tabletops is limited. 
Shape displays (e.g., [2]) create height maps over planar surfaces. Tactile displays enrich the limited haptic feedback of planar surfaces with local haptic stimuli (see [10] for an overview). A recent approach that changes the actual physical properties of a surface is MudPad [4]. By applying magnetic fields to a magnetorheological fluid in a planar pouch, the viscosity of the liquid and, thus, the stiffness of the surface can be changed locally.

Tangibles bring physical objects to interactive tabletops. They provide haptic feedback and are usually augmented with a visual projection. Several actuation techniques, e.g., using electromagnetic forces [8, 9, 15], vibration [12], or small motors [6], maintain the physical-visual consistency of the controls. We previously proposed to use electromagnetic fields that vary the resistance of controls, such as buttons and sliders [15]. This paper contributes empirical evidence for the non-trivial mapping of electromagnetic force to physical properties of tangible tabletop controls.

\section{INDUCING PHYSICAL EFFECTS BY MAGNETISM}

In this section, we describe how to use magnetism to dynamically change physical properties of passive tangibles. Our hardware is a smaller version of our Madgets actuation table [15] with a size of $25.2 \mathrm{~cm} \times 12.6 \mathrm{~cm}$, containing 72 electromagnets. All tangibles are crafted from acrylic using a laser cutter. Permanent magnets attached to the tangibles allow to apply forces using magnetic fields. Each electromagnet runs at $35 \mathrm{~V}$ and $0.25 \mathrm{~A}$; we regulate its power using pulse width modulation (PWM). In the following, we denote the strength of an electromagnet in percent, from $100 \%$ for a full power PWM linearly down to $0 \%$ (magnet deactivated).

\section{Weight}

Although weight is an inherent attribute of tangibles, electromagnetic actuation can be employed to change their perceived weight on the table by pulling one or more incorporated permanent magnets down with varying force. Applications can use the dynamic weight of a physical object to communicate its inner state in a haptic, eyes-free fashion. An example is a tangible file management application that uses tangibles to represent folders. In analogy to Auditory Icons [1], folder tangibles containing many or large files could be harder to move on the table than those with few or small files.

Our prototype consists of a box with attached magnets on the bottom plate. Without using actuation, raising the box away from the table requires the user to overcome the normal force that equals the weight of the box. We increase the normal force linearly by attracting the permanent magnets in this box, as our measurements reveal (Fig. 2). Since the magnetic force reduces quadratically in height, the effect of varying weight vanishes when the tangible is lifted. However, we can reliably manipulate the perceived weight for tangential motions on the table, as in our example above.

\section{Friction}

The friction of moveable parts in controls, such as knobs and sliders, determines the resistance against rotational or linear

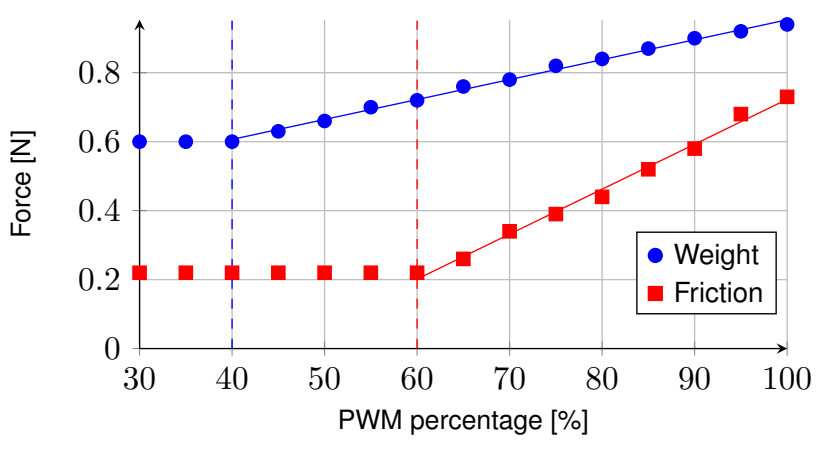

Figure 2. Weight and friction measurements. Since the permanent magnets also polarize the iron cores in the electromagnets, a minimum threshold of $40 \%$ is required to create a pulling force (weight). The minimum magnetic power to lift the brake block is $60 \%$ (friction).

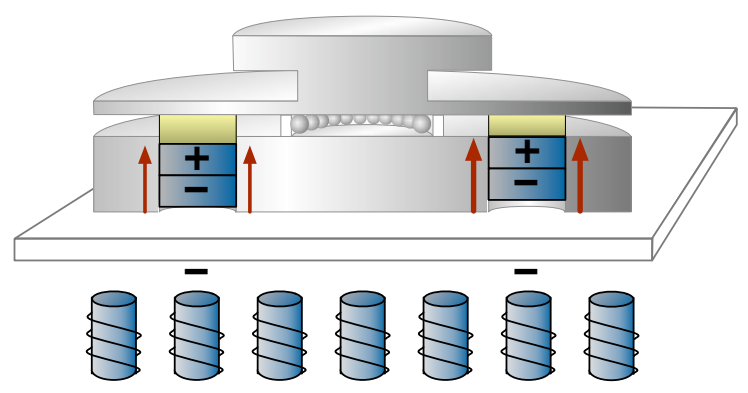

Figure 3. Cross-section of knob. Magnetic brake blocks are pushed against the rotary disk to change the perceived friction. The friction force induced by each block depends on the normal force (red) and on the materials of the brake facing (yellow) and the disk.

movements. Beyond the haptic sensation, the stiffness of a control also influences the user's perception of its function. As explained in [14], smooth-running knobs suggest uncritical values, while heavy knobs evoke the feeling of changing important data. Thus, by altering the friction in a control, the perception of its impact can be dynamically influenced. For example, a knob on a table controlling the temperature of an air condition could be smooth-running within a moderate range and stiff if a lower or upper threshold is exceeded.

We use a magnetically actuated disk brake to change the friction of a knob in real time (Fig. 3). By applying a repelling magnetic field, we push a permanent magnet with an attached brake block against the knob's disk - the stronger the applied electromagnetic field, the higher the friction in the knob. As friction also depends on the materials between the touching surfaces, our prototype knob contains multiple brakes with different materials that can be combined. Furthermore, brakes can be replaced quickly. Fig. 2 shows the measurements for a knob with a sandpaper brake pushing against a disk coated with velour. We achieve a nearly linear relation between PWM and measured friction force.

\section{Spring resistance}

Buttons usually contain a spring that raises a small plate to the top. Pushing down this plate to a pressure point triggers an event. Similar to friction, the resistance of a button can be 


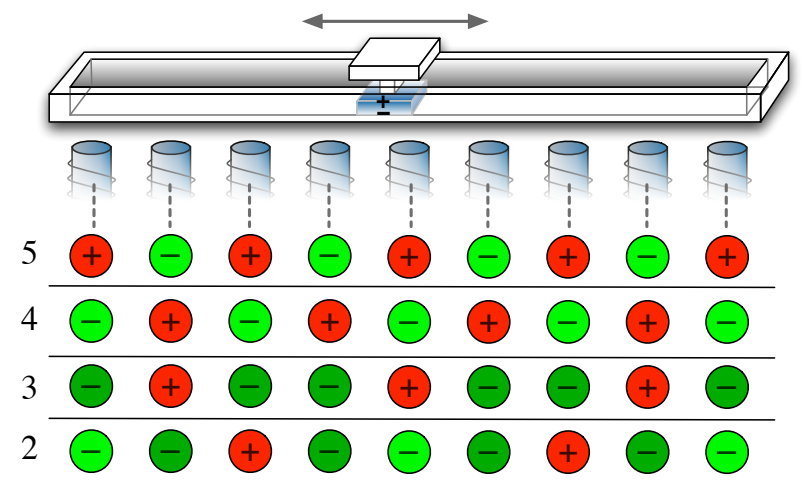

Figure 4. Slider with dynamic detents. Top: The knob handle is mounted on a permanent magnet that is influenced by nine electromagnets in the table. Bottom: Activation scheme of electromagnets to simulate 5, 4, 3, and 2 detents. Brightness represents power.

used for additional feedback. Hoffmann et al. [3] present a haptic keyboard that physically blocks individual keys while the user is typing, and report that increasing the stiffness of keys that are unlikely to be pressed next significantly reduces typing errors.

Buttons with dynamic spring resistance can easily be prototyped in our setup by adding small magnets to a button's plate (Fig. 1). Electromagnetic fields push the plate upwards, and the higher the applied power to the electromagnets, the higher the perceived resistance of the button is. As the magnetic force increases quadratically with height, it simulates a button containing a progressive spring. Our user tests in the next section examine the resolution of this control.

\section{Detents}

Detents discretize continuous controls, such as sliders and knobs, and constrain users to choose among a finite set of possible values. By mounting a permanent magnet on a control's handle, alternating electromagnetic fields can simulate detents by pulling the magnetic handle to the detent position (Fig. 4). Different polarizations of the electromagnets beneath the control yield a different number of detents. In our case, positively polarized electromagnets (red) attract the handle and, therefore, represent the detents. This effect is amplified by adjacent negatively polarized magnets (green) that push the handle away to the nearest detent when reaching nearby intermediate values.

Depending on the resolution of the magnetic array, the step size of the detents can be adapted dynamically. This allows to switch between continuous and discrete sliders, and to adapt the step size to different scales. Our slider prototype (Fig. 1) is $18 \mathrm{~cm}$ long and allows to switch the detent resolution between two and five steps. We evaluated how many detents users can distinguish with haptic feedback.

\section{USER TESTS}

We conducted two user tests to challenge the reproducibility of our approach. 11 participants ( 8 male, 3 female) aged 23 and $30(\mathrm{M}=26.27, \mathrm{SD}=2.45)$ conducted both tests in a single session. Tests were double blind, i.e., neither experi- menter nor participant could see the correct result in a trial. Results were stored in a database and evaluated after the test. Our test setup is shown in Fig. 1.

\section{Spring resistance}

In the first user test, we investigated whether we can replicate the spring resistance of buttons and which resolution we can achieve with pushing magnetic fields.

\section{Methodology}

As shown in Fig. 1, four prototype pushbuttons were placed on the table. All buttons were constructed in the same way using the same materials. During a trial, buttons were raised with different strengths. The participant was asked to sort these buttons by strength in descending order. She was allowed to push each button as often as needed. Accordingly, the experimenter entered her statement into a logging software. Each trial was interleaved with a short break.

We tested three conditions with increasing level of difficulty, beginning with two, then three, and then four buttons. We chose button strengths to start with a power that barely lifts the button up $(60 \%)$ and to end with the strongest possible power $(100 \%)$. Assuming a non-linear relation between the strengths of stimulus and perception (Weber-Fechner law), we used a scale factor that equalized the relative increase of strength from button to button. For the three-button condition we raised the buttons with $60 \%, 77.5 \%$, and $100 \%$ power, respectively; for the four button condition with $60 \%, 71.1 \%, 84.3 \%$, and $100 \%$, respectively.

Each condition contained ten trials; the order of button strengths was randomized. We measured the number of incorrectly detected sequences per condition and participant. We hypothesized that the participants could distinguish up to four different levels of rendered button resistances.

\section{Results}

No participant had any difficulties to distinguish the strength of two buttons (no errors). At the condition with three buttons, participants made 0.82 mistakes on average in ten trials $(\mathrm{SD}=0.98)$, with five participants making no mistake at all. At the four button condition, 1.64 incorrect sequences were reported on average $(\mathrm{SD}=1.57)$, including four persons who produced no errors.

\section{Discussion}

Our results show that electromagnetic actuation can simulate different resistance strengths of buttons. Errors were equally distributed among the conditions and, therefore, do not result from differences in the construction of the prototypes. The results show that participants can mostly distinguish four different strengths, with a higher error rate in the four button condition. The haptic resolution of such a pushbutton depends on several factors: a lighter button plate, a shorter actuation distance, and a higher voltage increase the force of repulsion, and, therefore, expand the range of possible resistance values. Furthermore, the permanent magnets should be chosen carefully. More magnets on the button plate cause a higher force of repulsion but also a higher weight. 


\section{Detents}

In our second experiment, we investigated the concept of electromagnetic detents in a continuous slider control.

\section{Methodology}

A slider control was mounted on the table (Fig. 1). For each trial, we applied different electromagnetic fields to simulate various detent configurations. A participant could shift the sliding knob to the left and the right as long as she wanted. After that, she was asked to report the number of detents she felt. The experimenter entered the data accordingly. During the test, the participant was not allowed to look at the control to ensure that she only used her sense of touch. She performed ten trials while we randomize the number of simulated detents between two and five in each trial.

\section{Results}

On average, test persons made 1.46 mistakes in ten trials (SD $=1.51$ ), with three participants detecting all detents. Four detents were recognized in all cases but one $(3.7 \%$ false rejects), followed by three and five detents $(11.5 \%$ and $11.1 \%$ false rejects, respectively). In the configuration involving two detents, nearly one third $(30.0 \%)$ of the decisions was incorrect.

\section{Discussion}

Our test shows that alternating polarization of adjacent magnets yields a suitable way to simulate detents. A positively polarized electromagnet next to one or two negative ones creates a strong force towards the detent position. While recognition rates are quite high for three to five detents, many participants had major difficulties to recognize the two detents configuration. In this case, the detent positions are about $8.4 \mathrm{~cm}$ away from each other. Between the detents, their pulling force is too low to overcome the friction and to attract the sliding knob. Accordingly, two adjacent detents should not span more than two magnets in such a setup. In this user test, our proof-of-concept prototype is aligned with the table's electromagnets. To allow detents in any orientation on the table, the density of magnets should be increased. Alternatively, dynamic magnetic fields can be used to simulate a higher magnet resolution. However, this requires tracking the sliding knob in real-time.

\section{CONCLUSION AND FUTURE WORK}

We applied electromagnetic actuation to render physical effects in tangible tabletop controls. Our studies show that electromagnetic force can be mapped to simulated properties, such as varying weight, friction, spring resistance, and dynamic detents. In future work, we will refine the hardware setup to achieve a higher output resolution. Furthermore, we intend to design and evaluate various applications that make use of dynamic physical effects in tabletop tangibles.

\section{ACKNOWLEDGEMENTS}

The German B-IT Foundation funded this work in part.

\section{REFERENCES}

1. W. W. Gaver. Auditory Icons: using sound in computer interfaces. Human-Computer Interaction, 2(2):167-177, 1986.

2. C. Harrison and S. E. Hudson. Providing dynamically changeable physical buttons on a visual display. In Proc. of CHI '09, 299-308.

3. A. Hoffmann, D. Spelmezan, and J. Borchers. TypeRight: a keyboard with tactile error prevention. In Proc. of CHI '09, 2265-2268.

4. Y. Jansen, T. Karrer, and J. Borchers. MudPad: tactile feedback and haptic texture overlay for touch surfaces. In Proc. of ITS '10, 11-14.

5. S. Jorda, G. Geiger, M. Alonso, and M. Kaltenbrunner. The reacTable: exploring the synergy between live music performance and tabletop tangible interfaces. In Proc. of TEI '07, 139-146.

6. A. Krzywinski, H. Mi, W. Chen, and M. Sugimoto. RoboTable: a tabletop framework for tangible interaction with robots in a mixed reality. In Proc. of ACE '09, 107-114.

7. T. H. Massie and K. J. Salisbury. The PHANToM haptic interface: a device for probing virtual objects. In Proc. of ASME '94, 295-302.

8. G. Pangaro, D. Maynes-Aminzade, and H. Ishii. The Actuated Workbench: computer-controlled actuation in tabletop tangible interfaces. In Proc. of UIST '02, 181-190.

9. J. Patten and H. Ishii. Mechanical constraints as computational constraints in tabletop tangible interfaces. In Proc. of CHI '07, 809-818.

10. I. Poupyrev, T. Nashida, and M. Okabe. Actuation and tangible user interfaces: the Vaucanson duck, robots, and shape displays. In Proc. of TEI '07, 205-212.

11. C. Ramstein and V. Hayward. The pantograph: a large workspace haptic device for multimodal human computer interaction. In Proc. of CHI '94, 57-58.

12. D. Reznik and J. Canny. C'mon part, do the local motion! In Proc. of ICRA '01, 2235-2242.

13. K. Salisbury, F. Conti, and F. Barbagli. Haptic rendering: introductory concepts. IEEE Computer Graphics and Applications, 24(2):24-32, 2004.

14. C. Swindells, K. E. MacLean, K. S. Booth, and M. J. Meitner. Exploring affective design for physical controls. In Proc. of CHI '07, 933-942.

15. M. Weiss, F. Schwarz, S. Jakubowski, and J. Borchers. Madgets: actuating widgets on interactive tabletops. In Proc. of UIST'10, 293-302.

16. M. Weiss, J. Wagner, Y. Jansen, R. Jennings, R. Khoshabeh, J. D. Hollan, and J. Borchers. SLAP widgets: bridging the gap between virtual and physical controls on tabletops. In Proc. of CHI '09, 481-490. 